\title{
Analyzing the effect of different types of Interline Power Flow Controllers
}

\author{
Y.N.Vijayakumar ${ }^{1}$, Dr. Sivanagaraju ${ }^{2}$ \\ Department of EEE, SVCET, Chittoor, A.P ${ }^{1}$ \\ Department of EEE, JNTUK, Kakinada, A.P ${ }^{2}$
}

\begin{abstract}
The latest development of Convertible Static Compensators (CSC) drives the importance of the Flexible AC Transmission System (FACTS) controllers to higher levels because of their controllable nature and effective power flow management of multi-line systems. There are several possibilities of operating configurations of FACTS devices by combing two or more converter blocks with flexibility. To realize the effect of CSC on a given power system, Interline Power Flow Controller (IPFC) is considered and Power Injection Model (PIM), Voltage Source Converter Model (VSM) and Hybrid Model for the same device is proposed in this thesis. The comfort in mathematical modeling and the incorporation procedure in Newton- Raphson load flow with these models increase its importance. Most of the literature concentrates on the voltage based controlled power flow using equivalent power injections. But the main contribution in this thesis is, to propose PIM, VSM and Hybrid models of IPFC to incorporate in NR load flow. By varying the device control parameters the variations in the system parameters is observed and are analyzed with the supporting results. Numerical results for HALE network and IEEE-30 bus systems without and with IPFC for the variation of device control parameters for each of the model are presented in detail. The obtained results show the performance of the proposed IPFC models.
\end{abstract}

Keywords: FACTS, IPFC, PIM, VSC, NR Load flow, IEEE - 30 Bus, MATLAB/SIMULINK

\section{INTRODUCTION}

An electrical power system can be seen as the interconnection of generating sources and customer loads through a network of transmission lines, transformers, and ancillary equipment. Its structure has many variations that are the result of a legacy of economic, political, engineering, and environmental decisions. Based on their structure, power system may be broadly classified into meshed and longitudinal systems. Meshed systems can be found in regions with a high population density and where it is possible to build power stations close to demand centers. Longitudinal systems are found in regions where large amount of power have to be transmitted over a long distances from power stations to load demand centers. Independent of structure of power system, the power flows throughout the network is largely distributed as a function of transmission line impedance. A transmission with low impedance enables large power flow through it than does transmission line with high impedance. This is not always the most desirable outcome because quite often it gives rise to a myriad of operational problems.

The operating problems are loss of system stability, power flow loops, high transmission losses, voltage limits violations, an inability to utilize transmission line capability up to thermal limit, and cascade tripping. Future, the expansion plan of many utilizes are being thwarted by a variety of well-founded, environmental, land-use, and regulatory pressures that prevent the licensing and building of new transmission lines and electricity generating plants overcome these problems. So a new solution to such operational problems will rely on the upgrading of existing transmission corridors by using recent power electronics controllers, known as Flexible Alternating Current Transmission Systems (FACTS) controllers. The salient feature of FACTS controller are fast response, direct power flow control through predetermined corridors, enhance stability and increase line loadings closer to the thermal limits. The power flow through the line can be changed by varying the voltages magnitude at the two ends, the phase angle or the line impedance.

To analyze the effect of FACTS controllers on a given system, we need help of modeling techniques to incorporate the device. In this project, the main aim is to develop a method to incorporate IPFC in a given system and identify the effect of the device in a less time by decreasing the complexity in programming code.

\section{INTERLINE POWER FLOW CONTROLLER}

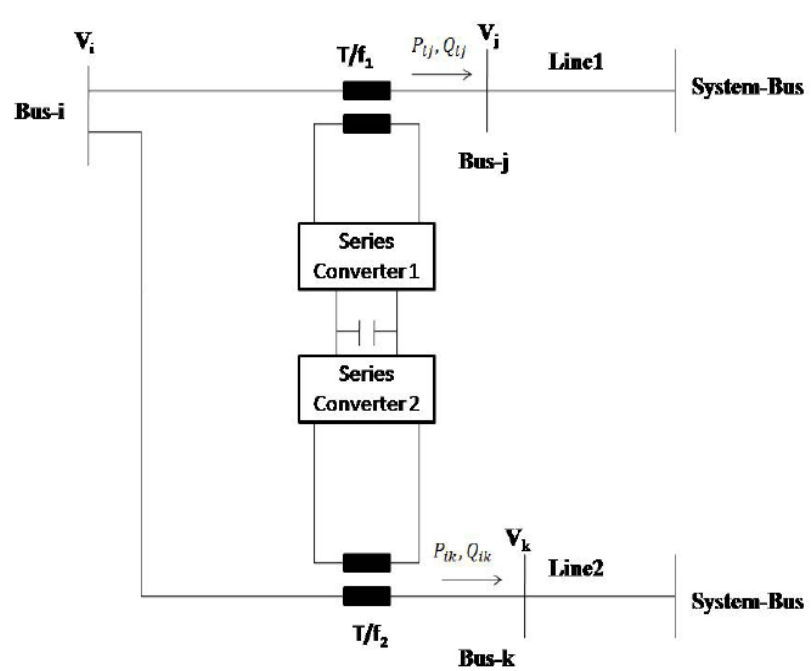

Fig. 1 Schematic diagram of two converters IPFC

An advanced and versatile member of FACTS controller is Interline Power Flow Controller. In general, IPFC is used in multiple transmission lines of a power system network. In its general form the interline power flow controller employs a number of dc-to-ac converters each providing series compensation for a different line. In other words, the IPFC 
comprises a number of static synchronous series compensators. The simplest IPFC consists of two back-to-back dc-to-dc converters, which are connected in series with two transmission lines through series coupling transformers and the dc terminals of the converters are connected together via a common dc link as shown in Fig. 1. In addition to providing series reactive compensation, any converter can be controller to supply real power to the common dc link from its own transmission line.

In order to select appropriate branch for placement of FACTS devices contingency analysis and sensitivity index method is used. Sensitivity index is introduced for ranking the optimal location. Sensitiveness of each branch towards the change in power transfer capability, voltage profile with respect to change in line reactance, reactive power injection at various branches, buses are identified. The sensitivity indexes at different branches have been evaluated to select appropriate branch for device location by using load flow technique. These sensitivity indexes reject how the load ability of branch change with respect to line reactance, how the voltage profile can be change with respect to reactive power injection at a particular bus. Sensitivity indices are evaluated for the base case and arrange in descending order. The branch which corresponds to highest sensitivity index is highest severe contingency.

$$
\text { Severity Index }(S I)=\sum_{i=1}^{n l}\left(\frac{S_{\text {flow }, i}}{S_{i}^{\max }}\right)^{2}
$$

Where $\mathrm{i}$ - branch number, $\mathrm{nl}$ - total number of lines

The power flows of highest severe contingency are considered as base case. In these flows the branch with least power flow can be considered as the best location for the IPFC device. Because the transmission line has inductive reactance where as the IPFC is a series controlled device which can provide a capacitive reactance so that the total reactance of the branch which leads to the increase of load ability of the line where the device has been located.

\section{CONVENTIONAL IPFC MODELING}

IPFC is modeled using two conventional modeling techniques and are listed below

1. Power Injection Model (PIM)

2. Voltage Source Converter based Model (VSM)

These models are used to set the control of power flows of multiple lines. Based on these models it is possible to estimate the IPFC control variables.

This focuses on the steady-state modeling of the IPFC for the implementation of the device in the conventional power flow program using injection power flow IPFC model. The injection power flow IPFC model is based on the representation of IPFC in steady-state conditions by two voltage sources each are in series with a certain reactance. A MATLAB conventional NR power flow program has been modified in order to incorporate the injection power IPFC model in power flow program.

An IPFC can be represented in steady-state conditions by two voltage sources representing fundamental components of output voltages of the two converters and impedances being the leakage reactance's of the two coupling transformers. Fig. 2 depicts a two voltage-source model of the IPFC. The two voltage sources, Vse are controllable in both magnitudes and phase angles and can be defined as:

$$
\bar{V}_{s e}=r V_{i} e^{j \gamma}
$$

The variable ' $r$ ' represents certain percent of the voltage magnitude $\mathrm{V}_{\mathrm{i}}$ at bus-i. The values of ' $\mathrm{r}$ ' and ' are defined within specified limits given as

$0 \leq r \leq r_{\max }$ and $0 \leq \gamma \leq \gamma_{\max }$

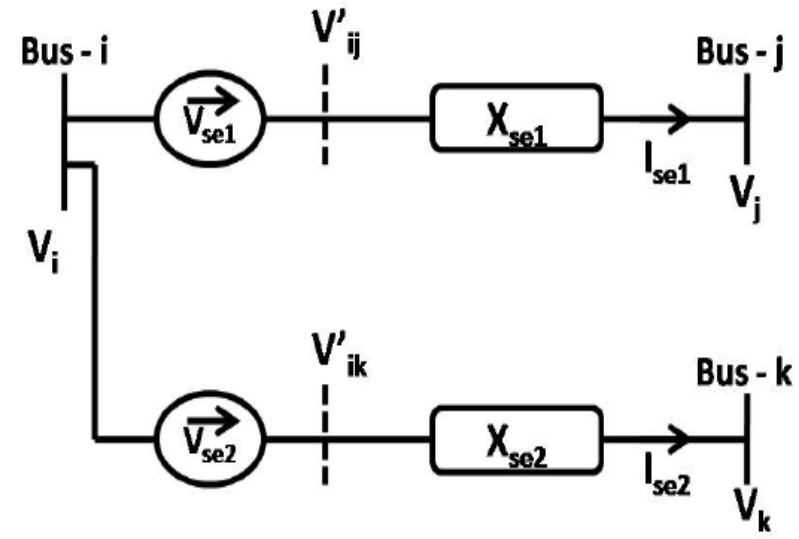

Fig. 2 Two series connected voltage source model

\section{VOLTAGE SOURCE CONVERTER BASED MODEL}

The voltage source converter model (VSM) represents the shunt and series VSCs directly as shunt and series voltage injections, respectively for IPFC. The VSMs of the FACTS controllers can be readily incorporated into the Newton- Raphson load flow algorithm, with the FACTS control variables as part of the expanded state variables. The mismatch equations are expanded to include the FACTS controller set point equations, such that the Jacobian matrix is also expanded as shown in Fig. 3.

With the Newton-Raphson solution technique, the VSM is capable of modeling various operating modes by substituting appropriate mismatch equations and by modifying the corresponding Jacobian matrix.
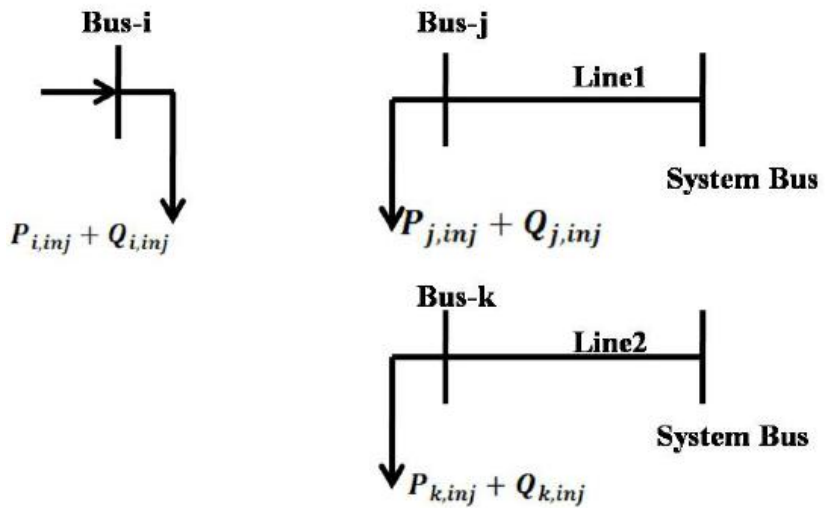

Fig. 3 Voltage Source Converter based IPFC model

The active and reactive power injections at IPFC buses i , j, $\mathrm{k}$ are

$P_{i, i n j}=V_{i} V_{s e_{i j}} B_{s e_{i j}} \sin \left(\delta_{i}-\delta_{s e_{i j}}\right)+V_{i} V_{s e_{i k}} B_{s e_{i k}} \sin \left(\delta_{i}-\delta_{s e_{i k}}\right)$

$Q_{i_{i n j}}=-V_{i} V_{s e_{i j}} B_{s e_{i j}} \cos \left(\delta_{i}-\delta_{s e_{i j}}\right)-V_{i} V_{s e_{i k}} B_{s e_{i k}} \cos \left(\delta_{i}-\delta_{s e_{i k}}\right)$

$P_{j, i n j}=-V_{j} V_{s e_{i j}} B_{s e_{i j}} \sin \left(\delta_{j}-\delta_{s e_{i j}}\right)$

$Q_{j, i n j}=V_{j} V_{s e_{i j}} B_{s e_{i j}} \cos \left(\delta_{j}-\delta_{s e_{i j}}\right)$

$P_{k_{, i n j}}=-V_{k} V_{s e_{i k}} B_{s e_{i k}} \sin \left(\delta_{k}-\delta_{s e_{i k}}\right)$

$Q_{k, i n j}=V_{k} V_{s e_{i k}} B_{s e_{i k}} \cos \left(\delta_{k}-\delta_{s e_{i k}}\right)$

The amount of active and reactive powers supplied by the two series converters is calculated as 
$P_{s e_{1}}=V_{i} V_{s e_{i j}} B_{s e_{i j}} \sin \left(\delta_{s e_{i j}}-\delta_{i}\right)+V_{j} V_{s e_{i j}} B_{s e_{i j}} \sin \left(\delta_{s e_{i j}}-\delta_{j}\right)$

$Q_{s e_{1}}=-V_{i} V_{s e_{i j}} B_{s e_{i j}} \cos \left(\delta_{s e_{i j}}-\delta_{i}\right)-V_{j} V_{s e_{i j}} B_{s e_{i j}} \cos \left(\delta_{s e_{i j}}-\delta_{j}\right)$

Similarly,

$$
\begin{aligned}
& P_{s e_{2}}=V_{i} V_{s e_{i k}} B_{s e_{i k}} \sin \left(\delta_{s e_{i k}}-\delta_{i}\right)+V_{k} V_{s e_{i k}} B_{s e_{i k}} \sin \left(\delta_{s e_{i k}}-\delta_{k}\right) \\
& Q_{s e_{2}}=-V_{i} V_{s e_{i k}} B_{s e_{i k}} \cos \left(\delta_{s e_{i k}}-\delta_{i}\right)-V_{k} V_{s e_{i k}} B_{s e_{i k}} \cos \left(\delta_{s e_{i k}}-\delta_{k}\right)
\end{aligned}
$$

\section{HYBRID IPFC MODEL}

It is generally known that the performance of voltage source converter (VSC) based Flexible AC Transmission System (FACTS) devices are superior to those of the thyristor based ones and as a result, lot of attention is being paid (both from academia and industry) for studying the behavior of these VSC based controllers. The family of VSC based FACTS devices comprises of the static compensator (STATCOM), the static synchronous series compensator (SSSC), the unified power flow controller (UPFC) and the interline power flow controller (IPFC). Among these four devices, UPFC and IPFC are more versatile as they can control multiple electrical quantities simultaneously which is not possible either by using a STATCOM or a SSSC. Therefore, in the coming days, especially in the light of deregulation, UPFC and IPFC are expected to play a very major role in power system control.

Now, for studying the behavior of IPFC, power flow solutions of networks incorporating IPFC is required. Although the issues pertaining to load flow modeling of UPFC have been studied quite extensively, relatively less attention has been paid in the literature for IPFC in this regard.

Excellent techniques for power flow modeling of IPFC are presented in Detail studies of these methodologies reveal that the complexities of the software codes for incorporating an IPFC in Newton power flow algorithm increase manifold due to the following major reasons.

1. Modification of the existing power flow codes is required to include the contributions from the IPFC series converters in the power injection equations of the buses concerned.

2. Development of new codes is required to account for the active power flow equation of the IPFC itself.

3. A number of new Jacobian sub blocks, which are exclusively related to the IPFC, are contained in the system Jacobian matrix. As a result, fresh codes have to be written to compute these new sub blocks.

To reduce the complexities of the software codes for implementing Newton power flow algorithm, in this project, an approach for an IPFC power flow model is proposed. In this model, an existing ' $n$ ' bus system incorporated with an IPFC with ' $\mathrm{p}$ ' series converters is transformed to an equivalent ' $\mathrm{n}+\mathrm{p}$ ' bus system, without the IPFC. As a result, the programming complexity reduces substantially because of the following reasons.

1. The power injection for the buses concerned can be computed in the proposed model using existing power flow codes, as it is devoid of contributions from any IPFC series converter.

2. In the proposed model, existing power flow codes can be used to compute the active power flow of the IPFC itself, which equals the sum of bus active power injections of additional load flow buses.

3. Only one major Jacobian sub block need to evaluated in the proposed model.

\section{MODELING PROCEDURE}

Fig. 4 shows an ' $n$ ' bus power system network in which 'p' series converters of a single IPFC are connected. Without loss of generality, it is assumed that the converters are connected between the buses ' $i-j$ ', $(i+1)-(j+1)^{\prime}$ and so on, up to between buses ' $(i+p-1)-(j+p-1)$ '. It is also further assumed that the $g^{\text {th }}$ converter is connected at the sending end (SE), i.e., at $\mathrm{g}^{\text {th }}$ bus of the corresponding transmission line. The equivalent circuit of Fig. 4 is shown in Fig. 5.

In Fig. 5, the IPFC is represented by 'p' (series) voltage sources. The voltage source (not shown) is in series with the impedance $\mathrm{Z}_{\text {seg }}$ and is connected in series with the $\mathrm{g}^{\text {th }}$ transmission line (which is represented by its equivalent-pi circuit).

The total current through the $\mathrm{g}^{\text {th }}\left(1_{-} \mathrm{g} \_\mathrm{p}\right)$ series coupling transformer is $I_{s e g}$, which consists of two parts: $I_{g}$ flowing in the line series impedance and $\mathrm{I}_{\mathrm{g} 0}$, the line charging current.

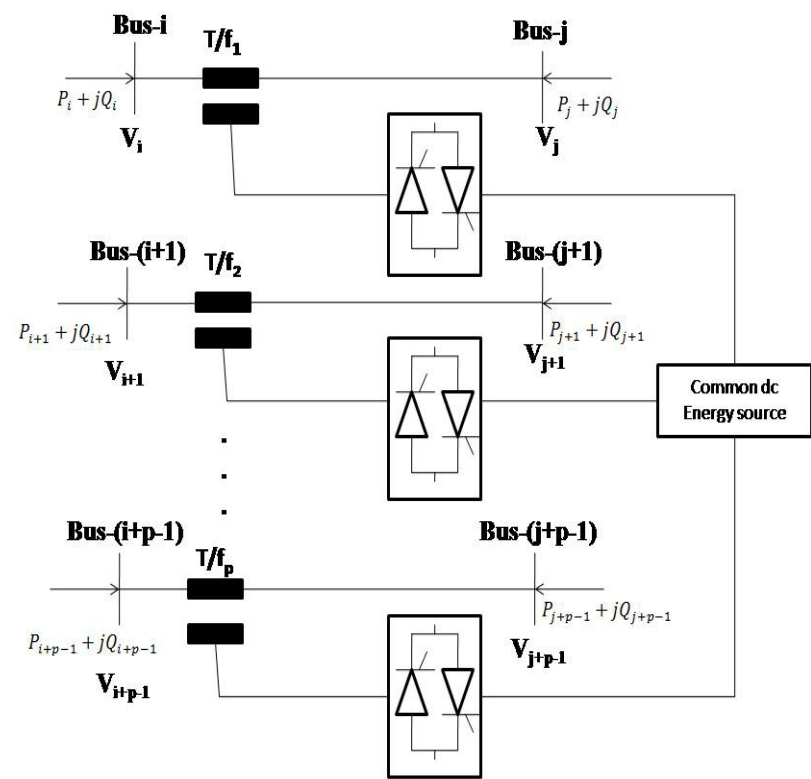

Fig. 4 IPFC with 'p' series converters connected to an existing ' $n$ ' bus
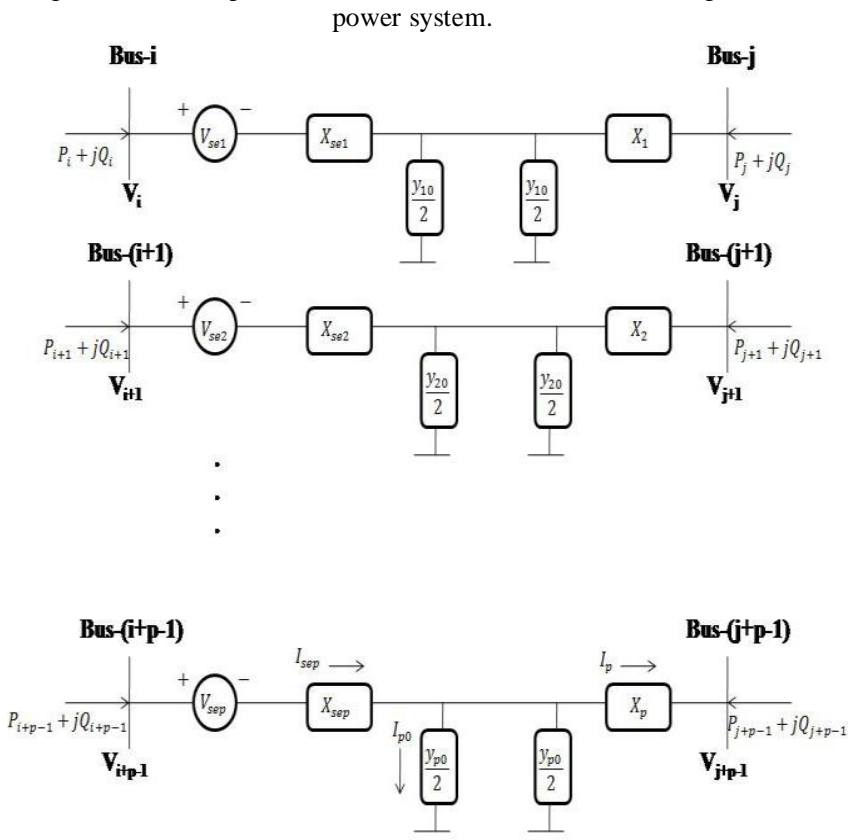

Fig. 5 Equivalent circuit of IPFC incorporated power system network. 
VII. RESULTS AND ANALYSIS

A. VSM of IPFC
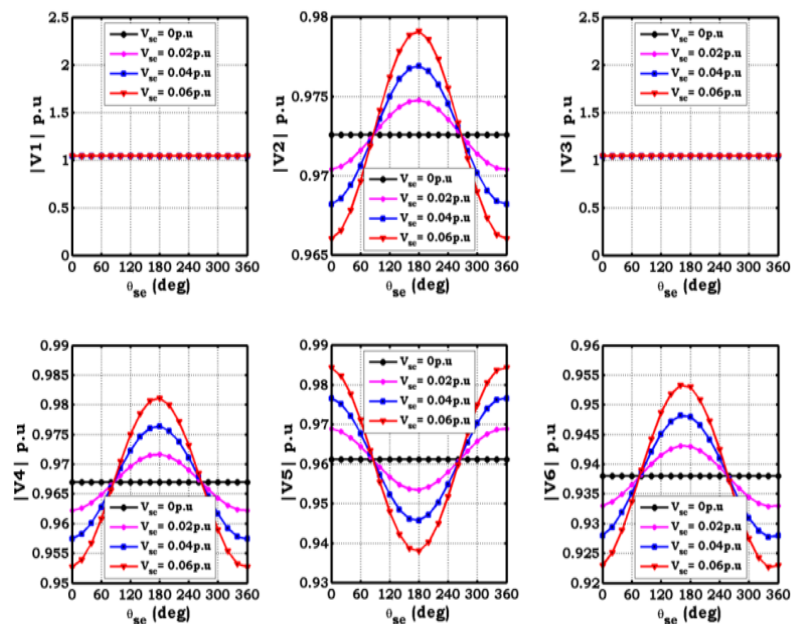

B.

PIM of IPFC
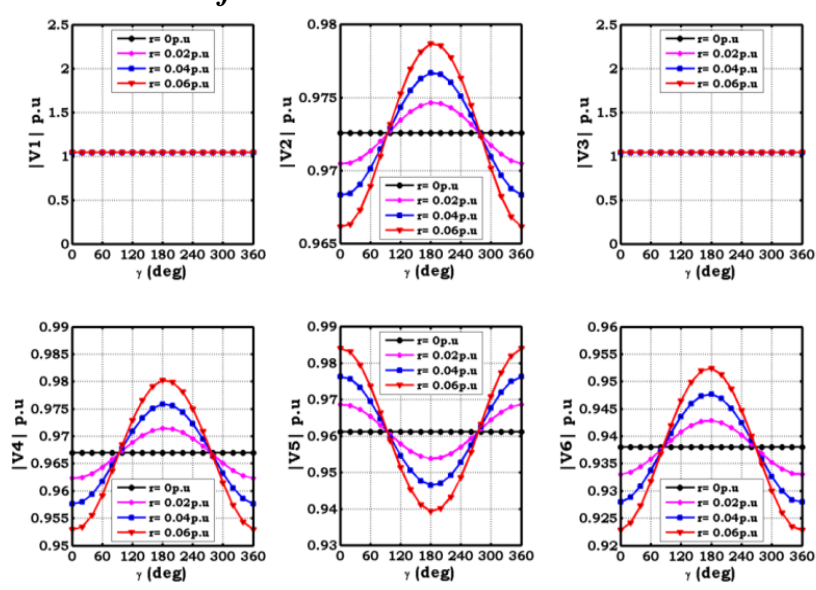

\section{CONCLUSION}

In this paper, the conventional models of IPFC like Power Injection Model (PIM) and Voltage Source Converter Models (VSM) are presented. The advanced model known as Hybrid Model is also analyzed and compared with the conventional models. The detailed mathematical modeling's of all these three models of IPFC with their incorporation procedures in conventional NR-load flow has been presented. These models are useful and effective as their modeling procedure was independent of the bus system.

Initially, for a given system, optimal location to install IPFC has been identified by calculating sensitivity index through contingency analysis. From this procedure the lines which has highest sensitivity index was selected as an optimal location. Using the incorporation procedure and modifications of the corresponding models are implemented to solve load flow. The variation of the system parameters like, bus voltage, lines active and reactive power flows, system active and reactive power losses are observed with the variation of the corresponding device control parameters. From this it is very clear that, there is an effect of IPFC on a given system with the proposed models.
Finally, the developed hybrid model of the device needs very less time when compared with the conventional models of IPFC and this model enhances the system parameters to higher values within less time.

\section{REFERENCES}

1. Edris A. "csc bolsters new york transmission system". IEEE Power Engineering Review, 21, Dec. 2001

2. Gyugyi. L. Hingorani N.G. understanding facts concepts and technology of flexible ac transmission systems". IEEE Press, 2000.

3. Gyugyi Kovalsky L. Edris A., Zelingher S. _squeezing more power from the grid". IEEE Power Engineering Review, 22, June 2002.

4. Gyugyi L._uni_ed power_ow concept for_exible ac transmission systems". IEEE Proceedings-C, 139, July 1992.

5. Fuerte-Esquivel C. Ambriz-Perez H., Acha E._advanced svc model for newton- raphson load flow and newton optimal power flow studies". IEEE Trans on power systems, 15, 129-136.

6. Faur Z. T. Canizares C. A. _analysis of svc and tcsc controllers in voltage collapse". IEEE Trans on power systems, 14, Feb. 1999.

7. Edris A. Gyugyi L., Rietman T.R. _the uni_ed power_ow controller: A new approach to power transmission control". IEEE Trans on power delivery, 10, April 1995.

8. Jenkins N. Arulampalam A., Ekanayake J.B._application study of a statcom with energy storage". IEE Proc. Gener., Transm., Distri., 150, May 2003. 